\title{
BMJ Open Patient characteristics of the Accident and Emergency Department of Kenyatta National Hospital, Nairobi, Kenya: a cross-sectional, prospective analysis
}

\author{
Justin Guy Myers, ${ }^{1}$ Katherine M Hunold, ${ }^{2}$ Karen Ekernas, ${ }^{3}$ Ali Wangara, ${ }^{4}$ \\ Alice Maingi, ${ }^{4}$ Vincent Mutiso, ${ }^{5}$ Stephen Dunlop, ${ }^{6}$ Ian B K Martin ${ }^{7}$
}

To cite: Myers JG, Hunold KM, Ekernas $\mathrm{K}$, et al. Patient characteristics of the Accident and Emergency Department of Kenyatta National Hospital, Nairobi, Kenya: a cross-sectional, prospective analysis. BMJ Open 2017;7:e014974. doi:10.1136/ bmjopen-2016-014974

- Prepublication history for this paper is available online. To view these files please visit the journal online (http://dx.doi org/10.1136/bmjopen-2016014974).

Received 2 August 2017 Accepted 10 August 2017

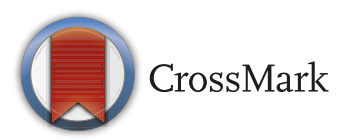

For numbered affiliations see end of article.

Correspondence to

Dr Justin Guy Myers;

justin_myers@med.unc.edu

\section{ABSTRACT}

Background Resource-limited settings are increasingly experiencing a 'triple burden' of disease, composed of trauma, non-communicable diseases (NCDs) and known communicable disease patterns. However, the epidemiology of acute and emergency care is not well characterised and this limits efforts to further develop emergency care capacity.

Objective To define the burden of disease by describing the patient population presenting to the Accident and Emergency Department (A\&E) at Kenyatta National Hospital (KNH) in Kenya.

Methods We completed a prospective descriptive assessment of patients in KNH's A\&E obtained via systematic sampling over 3 months. Research assistants collected data directly from patients and their charts. Chief complaint and diagnosis codes were grouped for analysis. Patient demographic characteristics were described using the mean and SD for age and $\mathrm{n}$ and percentages for categorical variables. International Classification of Disease 10 codes were categorised by 2013 Global Burden of Disease Study methods.

Results Data were collected prospectively on 402 patients with an average age of 36 years (SD 19), and of whom, $50 \%$ were female. Patients were most likely to arrive by taxi or bus $(39 \%)$, walking $(28 \%)$ or ambulance $(17 \%)$. Thirty-five per cent of patients were diagnosed with NCDs, $24 \%$ with injuries and $16 \%$ with communicable diseases, maternal and neonatal conditions. Overall, head injury was the single most common final diagnosis and occurred in $32(8 \%)$ patients. The most common patientreported mechanism for head injury was road traffic accident (39\%).

Conclusion This study estimates the characteristics of the A\&E population at a tertiary centre in Kenya and highlights the triple burden of disease. Our findings emphasise the need for further development of emergency care resources and training to better address patient needs in resourcelimited settings, such as $\mathrm{KNH}$.

\section{INTRODUCTION}

In low-income and middle-income countries (LMICs), stochastic infectious disease patterns are compounded by an increasing incidence
Strengths and limitations of this study

- Prospective and systematic sampling of Accident and Emergency Department patients via direct enrolment and chart review.

- This study highlights the growing burden of injuries and non-communicable diseases-compounding the existing burden of communicable conditions.

- One of the most common diagnoses and complaints, head injury, is elucidated by these findings.

- Results are grouped by the 2010 Global Burden of Disease study categories.

- Kenyatta National Hospital (KNH) is a tertiary referral hospital; therefore, the results may overestimate certain disease conditions in Kenya.

- Paediatric patients are underrepresented since KNH has a separate treatment area for non-traumatic patients 12 and under.

- Admission statistics may be underestimated as a result of difficulties in tracking patient flow in the A\&E.

of non-communicable diseases (NCDs) (such as heart disease and diabetes) and trauma (largely secondary to automotive accidents). This shifting epidemiology results in the well-described 'triple burden of disease': a baseline infectious disease burden with increasing NCD, and trauma, highlighted in the 2010 Global Burden of Disease (GBD) study. $^{12}$

Resource-limited health systems in East Africa have a reduced ability to care for patients presenting with acute care needs. ${ }^{3}$ Among their limitations is the widespread underdevelopment of emergency care systems. ${ }^{4} 5$ In most of Sub-Saharan Africa, Emergency Medicine (EM) is not a recognised specialty, ${ }^{6}$ and there is an associated absence of data describing patients presenting for acute, emergent and urgent care, as well as the overall burden of disease in this setting. Communicable diseases still 
receive a disproportionate share of health funding in LMICs, ${ }^{7-10}$ which is also attributable to a lack of baseline data, hindering development of trauma and cardiac resuscitative care systems. ${ }^{11}$ Resource-limited countries would benefit from a combination of disease surveillance, health workforce education and resource prioritisation to address all categories of disease, particularly as it relates to emergency care. ${ }^{12}$ Therefore, to continue to define the baseline burden of acute disease in this part of the world, we conducted a prospective, observational study, in the Accident and Emergency Department (A\&E) of Kenyatta National Hospital (KNH) in Nairobi, Kenya. The primary objective of our study was to characterise the presenting complaint, medical conditions, diagnoses and disposition of patients seen in the KNH A\&E.

\section{METHODS}

\section{Study setting and participants}

This is a single site study at the largest, public hospital in Kenya that is an 1800-bed tertiary care facility. Emergency services at KNH provide care in three areas: the A\&E, the Paediatric Emergency Unit (PEU) and the Outpatient Clinic. Providers in A\&E care for adult patients presenting with high-acuity medical and surgical conditions as well as severely injured adults and children. The PEU provides medical care to children aged $0-12$ years. Patients older than age 12 or injured (including burns) are directed to the A\&E. The annual patient census of these combined areas in 2014 was 163,426, with 69,294 patients treated in the A\&E (R Maina, personal communication). The A\&E is staffed by full-time and part-time medical officers and nurses, as well as nursing and emergency medical technician (EMT) students. Medical officers are physicians who have completed medical school and 1 year of internship. No medical officers have completed EM residency training; instead, they have varying expertise and postgraduate (consultant) training. Selected A\&E staff have completed the equivalent of Advanced Cardiac Life Support (ACLS), Advanced Trauma Life Support (ATLS) and possible disaster training. Many nurses have completed an additional 1 year emergency nursing certification course, a nursing training programme unique to $\mathrm{KNH}$.

All patients access care in the A\&E through a single triage point staffed by nurses, nursing students and/or EMT students. At triage, patient information, including chief complaint and limited vital signs are collected in paper logbooks; additional documentation is completed on one sheet of letter-sized paper. Patients are triaged as critical or non-critical. If critical, they are immediately directed to an acute resuscitation area in the A\&E for care. If non-critical, patients are directed either to the Outpatient Clinic, to the PEU (age 12 and under) or to the A\&E waiting area.

\section{Data collection}

Patients were enrolled in this study by a team of research assistants (RAs) via convenience and systematic sampling of every sixth patient registered in triage during 8 hour shifts from 11 March 2015 to 30 May 2015. If a sixth patient was not eligible or unavailable for the study, the RAs sought to enrol the very next (seventh) patient, and then resumed every sixth patient following that enrolment. Each RA enrolled patients approximately 40 hours per week and their 8-hour shifts included varying time frames across all 24 hours of the day and days of the week but were not randomised nor consecutive. This allowed a sampling of all time frames during the available working hours of the RAs.

All patients presenting to A\&E who could complete an informed written consent, or had next-of-kin available to consent, were eligible for this study. Patients were excluded if they could not consent and next-of-kin was not available, were unwilling, or were sent to a treatment area outside the A\&E. The first RA was placed in triage to identify every sixth patient. The second and third RAs completed consent, and collected initial information from the patient and chart throughout the visit. Because patient visits extended outside of data collection shift times, a fourth RA completed the data collection and, if admitted, tracked patients in the hospital. Data were entered via electronic tablets into REDCap, ${ }^{13}$ a secure, online database, supported by the University of Minnesota. ${ }^{14}$

Data collected included age, sex, chief complaint, mode of arrival, transfer status, acuity level, patient-reported trauma history, discharge diagnoses and disposition. If a patient reported a road traffic accident, he or she was asked if he or she was wearing a seatbelt. If a patient reported riding a motorcycle, he or she was asked about wearing a helmet. Patients were also given the opportunity to, but not required to, report their HIV status (if known).

Chief complaints and discharge diagnoses collected by RAs were assigned International Classification of Disease 10 (ICD-10) codes, as utilised in similar research settings. ${ }^{15} 16$ This was completed by specially trained RAs and classifications for both common and rare diagnoses were reached through consensus among the group and with the oversight by the principal investigator.

We aimed for a sample size that would be representative of the entire patient population. Our sample size $(n=402)$ was powered a priori to capture chief complaints/diagnoses that that occur in as few as $4 \%$ of the population with a precision of $\pm 2 \%$. Our sample provides an overview of common complaints at KNH over the specified time frame. We used the following formula for estimating the prevalence of disease in a standard population:

$$
\frac{\mathrm{n}=\mathrm{Z}^{2} \mathrm{P}(1-\mathrm{P})}{\mathrm{d}^{2}}
$$

where $n=$ sample size, $Z=Z$ statistic for level of confidence, $\mathrm{p}=$ expected prevalence or proportion and $\mathrm{d}=$ precision. 

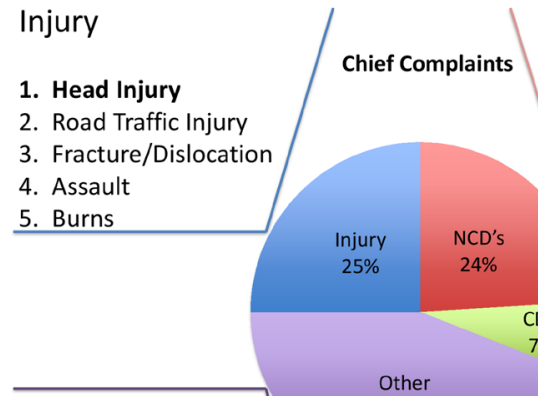

\section{Other \\ 1. Abdominal Pain \\ 2. Chest Pain \\ 3. Cough/Dyspnoea \\ 4. Nausea/Vomiting}

5. Headache

Figure 1 Top five Accident and Emergency Department chief complaints by each global burden of disease category. CD, communicable disease; NCD, noncommunicable disease; URI upper respiratoryinfection.

\section{Data analysis}

For descriptive purposes, chief complaint and diagnosis codes were grouped for analysis in the WHO's 21 chapter format. ${ }^{17}$ Additionally, we grouped codes in accordance with the 2010-2013 GBD studies. ${ }^{21819}$ This method partitions diagnoses in three main categories: (1) NCDs; (2) communicable diseases, maternal, and neonatal conditions (CD\&Ms) and (3) injuries. This provides a simple comparison between known overall global (and country-level) patterns of disease to diseases presenting acutely for care. To our knowledge, this has not been applied to the acute care setting prior to this study.

All data analysis was completed using STATA V.14 (Stata, College Station, Texas, USA) and mapping was completed using ArcGIS V.10.1 (Esri, Redlands, California, USA). The University of Nairobi/KNH Ethics Review Committee and the University of North Carolina

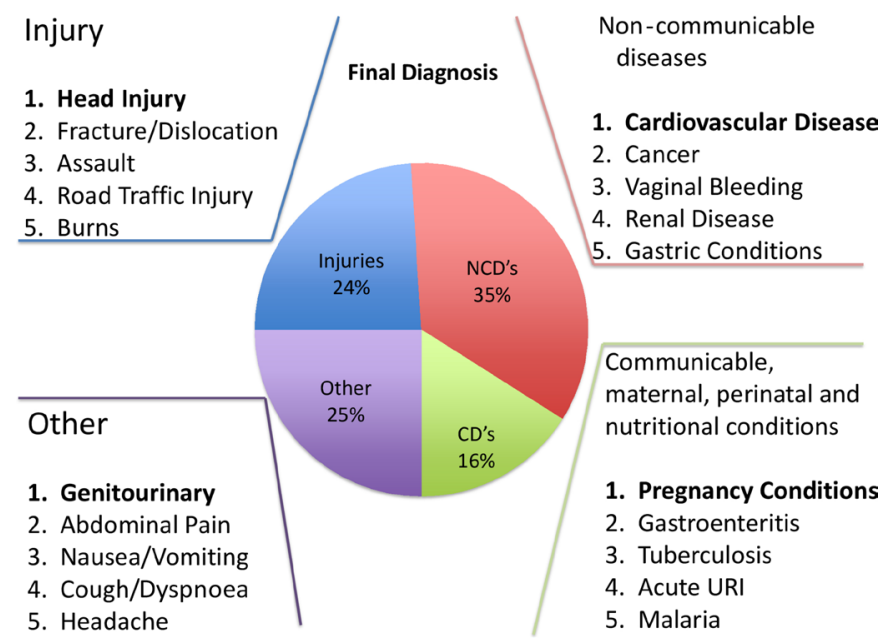

Figure 2 Top five Accident and Emergency Department final diagnoses by each global burden of disease category. CD, communicable disease; NCD, non-communicable disease; URI upper respiratoryinfection. at Chapel Hill Institutional Review Board approved the study.

\section{RESULTS}

The A\&E registered 14956 patients during the study period. ${ }^{13}$ During the RA shifts, 449 eligible patients were identified. Of these, 47 were excluded for a final sample size of 402. The number of eligible patients was less than every sixth patient as total RA enrolment hours in the A\&E were determined based on the sample size calculation. Importantly, final RA shifts encompassed all days of the week and all hours of the day. Reasons for exclusion included: eight $(17 \%)$ could not be consented due to a language barrier or were in critical condition and/or next of kin were unavailable, $10(21 \%)$ refused participation, two $(4 \%)$ were sent to labour and delivery, one (2\%) was sent to the PEU, three (6\%) were sent to other outpatient clinics and $26(55 \%)$ left the A\&E before consent.

Presenting patient chief complaints and subsequent final A\&E diagnoses were grouped according to three major categories of the GBD study. Among chief complaints, $25 \%$ were injuries, $24 \%$ were NCDs and $7 \%$ were communicable diseases and maternal or neonatal conditions (figure 1). Forty-four per cent were grouped as 'other' according to this methodology. A\&E final diagnoses were composed of $24 \%$ injuries, $35 \%$ NCDs and $16 \%$ were communicable diseases and maternal or neonatal conditions (figure 2). Twenty-five per cent were grouped 'other'. The top-five chief complaints and diagnoses are listed within each category in figures 1 and 2 .

The average age of participants was 36 years (SD 19 years), and patients were $50 \%$ female. Patients were most likely to arrive via taxi or bus $(39 \%)$, walking $(28 \%)$ or ambulance $(17 \%)$. During the study period, A\&E nursing were undergoing training on the South African Triage Scale (SATS), ${ }^{20}$ to replace the former gestalt method. Since the training was not completed at the time of data collection, trained RAs and the primary investigator used available patient information to estimate the triage level based on SATS methodology. Seven per cent of patients were triaged as 'emergent', $14 \%$ as 'very urgent' $35 \%$ as 'urgent', $35 \%$ as 'routine' and 9\% 'undesignated'. Referrals/transfers from outside health centres comprises $37 \%$ of subjects. The majority of patients $(62 \%)$ were discharged and $17 \%$ were admitted. One per cent was considered dead on arrival, $1 \%$ died in the A\&E, $4 \%$ left against medical advice or eloped, $1 \%$ were transferred to another hospital and $6 \%$ of patients underwent a medical 'review' or were evaluated by a subspecialist in the A\&E. The remaining $10 \%$ of patients had missing or incomplete information on their chart, and the RAs

iPatients presenting for a medical 'review' usually present with test results from a clinic or outside hospital for subspecialty evaluation. RAs were unable to ascertain the exact disposition of this entire category of patients but noted these patients were likely discharged and not found in the admission logbook. 
Open Access

Table 1 Characteristics of patient population by age group. All data are presented as $n(\%)$ unless otherwise noted

\begin{tabular}{|c|c|c|c|c|c|}
\hline Characteristic & $\begin{array}{l}\text { All patients } \\
(\mathrm{n}=402)\end{array}$ & $\begin{array}{l}<5 \text { years } \\
(n=22)\end{array}$ & $\begin{array}{l}\leq 17 \text { years } \\
(n=48)\end{array}$ & $\begin{array}{l}\text { 18-64 years } \\
(\mathrm{n}=302)\end{array}$ & $\begin{array}{l}\geq 65 \text { years } \\
(n=44)\end{array}$ \\
\hline Age, mean $(S D)(n=394)$ & $36(19)$ & $1(2)$ & $7(6)$ & $35(12)$ & $75(7)$ \\
\hline \multicolumn{6}{|l|}{ Sex } \\
\hline Male & $201(50)$ & $12(55)$ & $29(60)$ & $146(48)$ & $23(52)$ \\
\hline Female & $201(50)$ & $10(45)$ & $19(40)$ & $156(52)$ & $21(48)$ \\
\hline \multicolumn{6}{|l|}{ Mode of arrival } \\
\hline Private vehicle & $16(4)$ & $0(0)$ & $0(0)$ & $12(4)$ & $4(9)$ \\
\hline Taxi/bus & $156(39)$ & $8(36)$ & $19(40)$ & $114(38)$ & $21(48)$ \\
\hline Ambulance & $69(17)$ & $5(23)$ & $7(15)$ & $53(18)$ & $5(11)$ \\
\hline Walked & $113(28)$ & $0(0)$ & $8(17)$ & $96(32)$ & $8(18)$ \\
\hline Carried & $30(8)$ & $8(36)$ & $12(25)$ & $16(5)$ & $2(5)$ \\
\hline Wheelchair & $10(3)$ & $0(0)$ & $1(2)$ & $4(1)$ & $4(9)$ \\
\hline Unknown/blank & $5(1)$ & $1(5)$ & $1(2)$ & $4(1)$ & $0(0)$ \\
\hline \multicolumn{6}{|l|}{ Transferred } \\
\hline Yes & $147(37)$ & $13(59)$ & $20(43)$ & $104(35)$ & $20(45)$ \\
\hline No & $238(60)$ & $8(36)$ & $25(53)$ & $188(63)$ & $21(48)$ \\
\hline Unknown & $13(3)$ & $1(5)$ & $2(4)$ & $7(2)$ & $3(7)$ \\
\hline \multicolumn{6}{|l|}{ Acuity level (estimated) $^{*}$} \\
\hline Emergency (red) & $29(7)$ & $3(14)$ & $5(11)$ & $20(7)$ & $4(9)$ \\
\hline Very urgent (orange) & $55(14)$ & $4(18)$ & $11(23)$ & $39(13)$ & $4(9)$ \\
\hline Urgent (yellow) & $140(35)$ & $8(36)$ & $12(26)$ & $107(35)$ & $18(41)$ \\
\hline Routine & $141(35)$ & $3(14)$ & $14(30)$ & $110(37)$ & $14(32)$ \\
\hline Not assigned/unknown/other & $36(9)$ & $4(18)$ & $5(11)$ & $26(9)$ & $4(9)$ \\
\hline \multicolumn{6}{|l|}{ Trauma history $\dagger$} \\
\hline Any & $98(24)$ & $8(36)$ & $20(42)$ & $72(24)$ & $4(9)$ \\
\hline Road traffic accident & $32(8)$ & $1(5)$ & $3(6)$ & $28(9)$ & $1(3)$ \\
\hline Motor or bicyclist & $5(1)$ & $0(0)$ & $0(0)$ & $3(1)$ & $2(5)$ \\
\hline Assault & $27(7)$ & $0(0)$ & $3(6)$ & $23(8)$ & $0(0)$ \\
\hline Pedestrian & $1(0)$ & $0(0)$ & $0(0)$ & $1(0)$ & $0(0)$ \\
\hline Burn & $8(2)$ & $3(14)$ & $5(10)$ & $3(1)$ & $0(0)$ \\
\hline Self-inflicted & $4(1)$ & $2(9)$ & $2(4)$ & $2(1)$ & $0(0)$ \\
\hline Fall & $21(5)$ & $2(9)$ & $7(15)$ & $12(4)$ & $1(2)$ \\
\hline \multicolumn{6}{|l|}{ Disposition } \\
\hline Discharge from A\&E & $250(62)$ & $13(59)$ & $25(52)$ & $192(64)$ & $30(68)$ \\
\hline Admit to hospital & $67(17)$ & $5(23)$ & $10(21)$ & $45(15)$ & $8(18)$ \\
\hline DOA & $2(1)$ & $0(0)$ & $0(0)$ & $2(1)$ & $0(0)$ \\
\hline Death in A\&E & $2(1)$ & $0(0)$ & $0(0)$ & $1(0)$ & $1(2)$ \\
\hline DAMA/eloped & $15(4)$ & $0(0)$ & $1(2)$ & $13(4)$ & $1(2)$ \\
\hline Transfer to outside hospital & $2(1)$ & $0(0)$ & $0(0)$ & $2(1)$ & $0(0)$ \\
\hline Consultant 'review' & $25(6)$ & $1(5)$ & $5(10)$ & $18(6)$ & 2 (5) \\
\hline $\begin{array}{l}\text { 'Missing/incomplete' or disposition field } \\
\text { blank }\end{array}$ & 39 (10) & 3 (14) & 7 (15) & 29 (10) & 2 (5) \\
\hline
\end{tabular}

${ }^{*}$ Determined by research assistants and/or primary investigator utilising the South African Triage Scale(SATS). ${ }^{20}$

†Patient reported.

A\&E, accident and emergency department; DOA, death on arrival; DAMA, discharged against medical advice. 


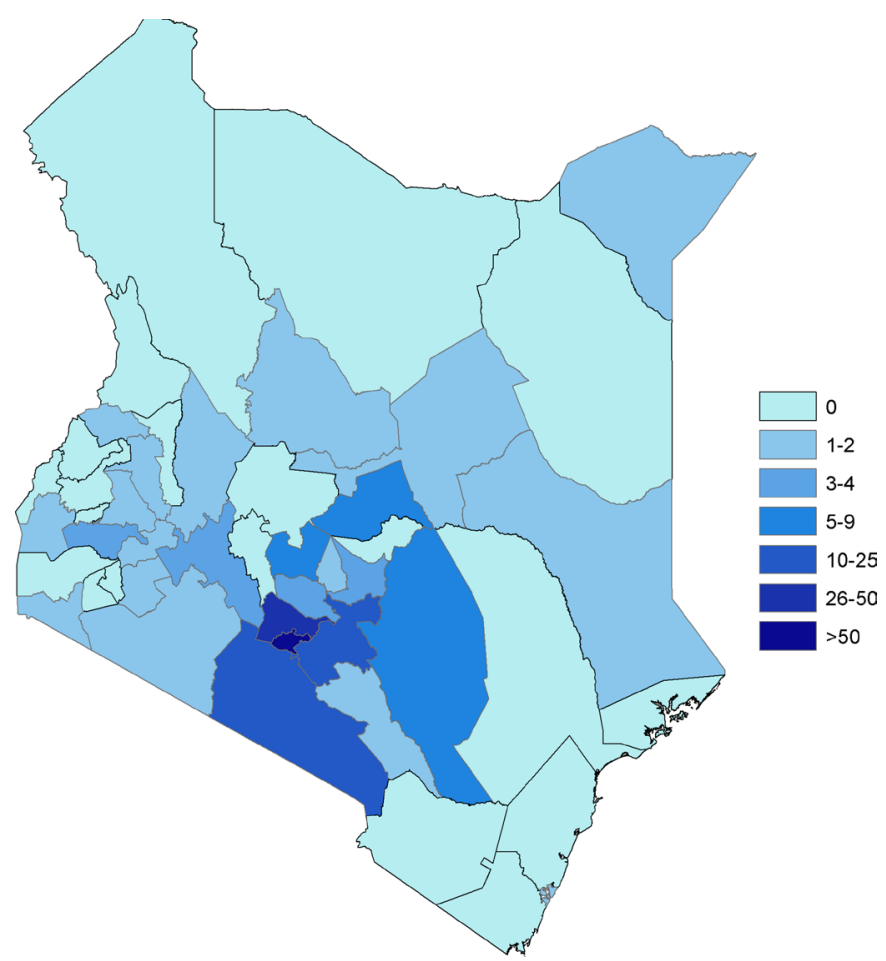

Figure 3 Kenya providence of origin of study patient population.

were unable to ascertain their disposition (table 1). The majority of patients were from Nairobi proper (figure 3).

Overall, abdominal pain was the single most common chief complaint and occurred in $47(12 \%)$ of patients. The most common WHO chapter was injury (table 2). Head injury was the single most common final diagnosis and occurred in $32(8 \%)$ patients. The most common patient-reported mechanism for head injury was road traffic injury (RTI) $(39 \%)$, followed by assault (36\%) and fall $(9 \%)$ (table 3$)$.

Forty-three per cent of participants reported a negative HIV status. Comparatively, $2 \%$ reported being HIV positive, $47 \%$ reported an 'unknown' status and $8 \%$ declined to respond.

\section{DISCUSSION}

This study provides an important overview of patients presenting for emergency care in Kenya. Wachira et al characterised patients presenting to a variety of A\&Es across Kenya over a single 24 hours time period and found that trauma, respiratory tract infections and malaria were the leading diagnoses. ${ }^{21}$ House et al conducted a more comprehensive analysis of A\&E care by retrospectively analysing all patients over a 1-year period at Moi Teaching and Referral Hospital, a public tertiary hospital in western Kenya. The top three A\&E diagnoses were injuries, infections and mental health disorders. ${ }^{16}$ Additional studies have focused on patients with acute coronary syndrome ${ }^{22}$ and emergency obstetrical care. ${ }^{23}$ Our results demonstrate a 'triple burden' of acute disease in the KNH A\&E of NCDs; communicable diseases, maternal and neonatal
Table 2 Final diagnosis among patient population. All data are presented at $\mathrm{n}(\%) ;(\mathrm{n}=402)$

\begin{tabular}{ll}
\hline $\begin{array}{l}\text { WHO International Classification of } \\
\text { Disease 10 chapter* }\end{array}$ & $\begin{array}{l}\text { Final } \\
\text { diagnosis } \\
\mathbf{n}(\%)\end{array}$ \\
\hline Injury & $92(23)$ \\
\hline Signs and symptoms & $55(14)$ \\
\hline Genitourinary system & $54(13)$ \\
\hline Circulatory & $39(10)$ \\
\hline Injury mechanism & $38(9)$ \\
\hline Digestive system & $34(8)$ \\
\hline Infectious and parasitic & $30(7)$ \\
\hline Neoplasms & $23(6)$ \\
\hline Musculoskeletal system & $22(5)$ \\
\hline Pregnancy, childbirth and the puerperium & $18(4)$ \\
\hline Respiratory & $16(4)$ \\
\hline Factors influencing health status & $15(4)$ \\
\hline Skin and subcutaneous tissue & $12(3)$ \\
\hline Diseases of blood & $9(2)$ \\
\hline Endocrine, nutritional and metabolic & $6(1)$ \\
\hline Nervous system & $6(1)$ \\
\hline Mental and behavioural & $5(1)$ \\
\hline Congenital/chromosomal & $4(1)$ \\
\hline Eye and adnexa & $3(1)$ \\
\hline Perinatal period & $2(1)$ \\
\hline Ear and mastoid process & $1(0)$ \\
\hline
\end{tabular}

${ }^{*}$ Chapters are not mutually exclusive.

conditions (CD\&Ms) and injuries, similar to the previously described triple burden of disease that is affecting Sub-Saharan Africa. ${ }^{124}$

In Kenya, specifically, CD\&Ms, NCDs and injuries rank first, second and third, respectively, for premature death (Years of Life Lost). Overwhelmingly, in 2015, CD\&Ms were responsible for the most causes of death, premature death and disability. ${ }^{25}$ However, our study demonstrates the opposite patterns of disease presenting for acute care; CDs were the most common cause, followed by injuries, with the least presentations for CD\&Ms.

\section{Non-communicable diseases}

NCDs account for $24 \%$ of chief complaints and $35 \%$ of A\&E diagnoses. Among NCDs, cardiovascular disease was the most common condition and the second most common of chief complaints. In the GBD study, Lozano et al found that NCDs, such as ischaemic heart disease, have become the greatest burden of disease worldwide, in terms of years of life lost (YLL). ${ }^{2}$ The same study reports that in Eastern Africa, ischaemic heart disease ranks \#17 in YLL, and unfortunately, age-matched death rates due to NCDs are higher in LMICs. ${ }^{26}$ Addressing this burden is challenging since global health initiatives and media coverage (eg, of the Ebola virus and Zika virus crises, etc) 
Table 3 Characteristics of patient population with head injury as defined by WHO International Classification of Disease 10 code block 'injury to the head' by age group. All data are presented as $n(\%)$ unless otherwise noted.

\begin{tabular}{|c|c|c|c|c|c|}
\hline Characteristic & All patients $(n=33)$ & $<5$ years $(n=2)$ & $\leq 17$ years $(n=5)$ & 18-64 years $(n=28)$ & $\geq 65$ years $(n=0)$ \\
\hline Age, mean (SD) & $29(14)$ & $3(1)$ & $5(2)$ & $33(10)$ & \\
\hline \multicolumn{6}{|l|}{ Sex } \\
\hline Male & $28(85)$ & $2(100)$ & $4(80)$ & $24(86)$ & \\
\hline Female & $5(15)$ & $0(0)$ & $1(20)$ & $4(14)$ & \\
\hline \multicolumn{6}{|l|}{ Mode of arrival } \\
\hline Private vehicle & $1(3)$ & $0(0)$ & $0(0)$ & $1(4)$ & \\
\hline Taxi/bus & $8(24)$ & $1(50)$ & $2(40)$ & $6(21)$ & \\
\hline Ambulance & $12(36)$ & $1(50)$ & $1(20)$ & $11(39)$ & \\
\hline Walked & $6(18)$ & $0(0)$ & $0(0)$ & $6(21)$ & \\
\hline Carried & $3(9)$ & $0(0)$ & $2(40)$ & $1(4)$ & \\
\hline Wheelchair & $1(3)$ & $0(0)$ & $0(0)$ & $1(4)$ & \\
\hline Unknown/blank & $2(6)$ & $0(0)$ & $0(0)$ & $2(7)$ & \\
\hline \multicolumn{6}{|l|}{ Transferred } \\
\hline Yes & $13(39)$ & $2(100)$ & $2(40)$ & $11(39)$ & \\
\hline No & $20(61)$ & $0(0)$ & $3(60)$ & $17(61)$ & \\
\hline Unknown & $0(0)$ & $0(0)$ & $0(0)$ & $0(0)$ & \\
\hline \multicolumn{6}{|l|}{ Acuity level (estimated)* } \\
\hline Emergency (red) & $4(12)$ & $0(0)$ & $1(20)$ & $3(11)$ & \\
\hline Very urgent (orange) & $8(24)$ & $1(50)$ & $2(40)$ & $6(21)$ & \\
\hline Urgent (yellow) & $12(36)$ & $0(0)$ & $0(0)$ & $12(43)$ & \\
\hline Routine & $7(21)$ & $1(50)$ & $1(20)$ & $6(21)$ & \\
\hline Not assigned/unknown/other & $2(6)$ & $0(0)$ & $1(20)$ & $1(4)$ & \\
\hline \multicolumn{6}{|l|}{ Trauma history $\dagger$} \\
\hline Any & $31(94)$ & $1(50)$ & $4(80)$ & $27(96)$ & \\
\hline Road traffic accident & $13(39)$ & $1(50)$ & $2(40)$ & $11(39)$ & \\
\hline \multicolumn{6}{|l|}{ Wearing seatbelt } \\
\hline Yes & $0(0)$ & $0(0)$ & $0(0)$ & $0(0)$ & \\
\hline No & $4(31)$ & $0(0)$ & $0(0)$ & $4(36)$ & \\
\hline Unknown & $7(54)$ & $1(100)$ & $2(100)$ & $5(45)$ & \\
\hline Not recorded & $2(15)$ & $0(0)$ & $0(0)$ & $2(18)$ & \\
\hline Motor or bicyclist & $1(3)$ & $0(0)$ & $0(0)$ & $1(4)$ & \\
\hline \multicolumn{6}{|l|}{ Wearing helmet $(n=1)$} \\
\hline Yes & $0(0)$ & $0(0)$ & $0(0)$ & $0(0)$ & \\
\hline No & $0(0)$ & $0(0)$ & $0(0)$ & $0(0)$ & \\
\hline Unknown & $1(100)$ & $0(0)$ & $0(0)$ & $1(100)$ & \\
\hline Assault & $12(36)$ & $0(0)$ & $0(0)$ & $12(43)$ & \\
\hline Pedestrian & $1(3)$ & $0(0)$ & $0(0)$ & $1(4)$ & \\
\hline Burn & $0(0)$ & $0(0)$ & $0(0)$ & $0(0)$ & \\
\hline Self-inflicted & $1(3)$ & $0(0)$ & $0(0)$ & $1(4)$ & \\
\hline Fall & $3(9)$ & $0(0)$ & $2(40)$ & $1(4)$ & \\
\hline \multicolumn{6}{|l|}{ Disposition } \\
\hline Discharge from A\&E & $19(58)$ & $1(50)$ & $3(60)$ & $16(57)$ & \\
\hline Admit to hospital & $5(15)$ & $1(50)$ & $1(20)$ & $5(18)$ & \\
\hline $\mathrm{DOA}$ & $0(0)$ & $0(0)$ & $0(0)$ & $0(0)$ & \\
\hline
\end{tabular}


Table 3 Continued

\begin{tabular}{lllll}
\hline Characteristic & All patients $(\mathbf{n}=\mathbf{3 3})$ & $<\mathbf{5 y e a r s}(\mathbf{n}=\mathbf{2})$ & $\mathbf{5 1 7}$ years $(\mathbf{n}=\mathbf{5})$ & $\mathbf{1 8 - 6 4}$ years $(\mathbf{n = 2 8}) \quad \mathbf{6 5}$ years $(\mathbf{n}=\mathbf{0})$ \\
\hline Death in A\&E & $1(3)$ & $0(0)$ & $0(0)$ & $1(4)$ \\
DAMA/eloped & $1(3)$ & $0(0)$ & $0(0)$ & $1(4)$ \\
Transfer to outside hospital & $1(3)$ & $0(0)$ & $0(0)$ & $1(4)$ \\
$\begin{array}{l}\text { Consultant 'Review' } \\
\text { 'Missing/incomplete' or }\end{array}$ & $3(9)$ & $0(0)$ & $1(20)$ & $2(7)$ \\
disposition field blank & $2(6)$ & $0(0)$ & $0(0)$ & \\
\hline
\end{tabular}

${ }^{*}$ Determined by research assistants and/or primary investigator utilising the South African Triage Scale(SATS) ${ }^{20}$

†Collected by research assistants. Categories are not mutually exclusive.

A\&E, accident and emergency department; DOA, death on arrival; DAMA, discharged against medical advice.

have typically prioritised important infectious diseases ${ }^{27-29}$ which may be influencing public perception ${ }^{30}$ and funding priorities. There is a need for preventive efforts and funding for improving acute care systems in Kenya to respond to the growing burden of NCDs.

\section{Injuries}

Injuries account for approximately one-quarter of both chief complaints $(25 \%)$ and final diagnoses $(24 \%)$. Head injury was by far the most common-comprising $26 \%$ of injury diagnoses and affecting $8 \%$ of all patients. The most common reported mechanism of head injury was RTI (39\%). A similar trend was found at Moi Teaching and Referral Hospital in Western Kenya, where RTI was the most common chief complaint, and head injury was in the top five diagnoses. ${ }^{16}$ Additionally, in a study from Kampala, Uganda (neighbouring Kenya), RTIs were the most common causes of injury for all age groups and $75 \%$ of admitted trauma mortality had concomitant head injury. ${ }^{31}$ Traumatic brain injury (TBI), most commonly caused by RTIs is likely underreported in Africa, due to poor access to care and absence of injury surveillance. ${ }^{32}$ The international collaborative trauma study, Clinical Randomisation of an Antifibrinolytic in Significant Haemorrhage (CRASH) predicts that patients with TBIin LMICs have over twice the odds of dying following severe TBI (OR 2.23, $95 \%$ CI 1.51 to 3.30$).{ }^{33}$ The high incidence of head injuries related to road traffic crashes in Kenya should raise concern.

Studies from LMICs demonstrate that legislative-based interventions are the single most effective strategy for reducing road traffic injuries and death due to RTIs. ${ }^{34}$ Of the 14 patients with head injuries from RTIs in our study, none reported use of seatbelts and/or helmets. This is lower than previously reported by Saidi et al in 2014 in admitted trauma patients at $\mathrm{KNH}$, where $11.6 \%$ of occupants wore seatbelts and $18.2 \%$ of motorcycle passengers wore helmets. ${ }^{35}$ This discrepancy may be due to the relatively low number of patients in this category and reliance on patient reporting. While motorcycle helmet, seat-belt and drunk driving laws exist in Kenya, they are poorly enforced. ${ }^{36}$ In 2010, a consortium including the WHO, Johns Hopkins University (JHU), and local Kenyan partners launched the Road Safety in 10 Countries (RS-10) campaign. This programme aims to reduce mortality from RTIs through social marketing, strengthening public health legislation, training local law enforcement and improving trauma care and data surveillance systems. ${ }^{37}$ This multifaceted approach holds promise, as there has been a modest increase in helmet utilisation and decrease in road speeds as a result. ${ }^{38}$

\section{Communicable, maternal, perinatal and nutritional disease}

The third major GBD study category, infectious diseases and maternal or neonatal conditions, appears to comprise a lesser share of the total burden of acute disease at $\mathrm{KNH}$, comprising only $16 \%$ of final diagnoses. This is curious, given that top five causes of death in this category in Kenya are from HIV/AIDS, diarrhoea, lower respiratory tract infections, neonatal encephalopathy, preterm births. ${ }^{25}$

The contribution of chronic infectious diseases, such as HIV, on acute health conditions is not well elucidated by this study. The HIV prevalence in this study was $2 \%$ (self-reported), which is lower than the documented HIV prevalence among adults aged 15-49 years in Kenya in 2014 of $5.3 \% .^{39}$ However, almost half $(47 \%)$ of patients in our study reported an unknown HIV status. Therefore, it is possible that there is a hidden HIV burden among patients at KNH's A\&E, who largely originate from Nairobi's large urban areas. HIV testing in the Emergency Department is well studied and practised ${ }^{4041}$ and is crucial for vulnerable populations, whose only access to healthcare may be the Emergency Department. ${ }^{42}$ As such, the A\&E represents an opportunity for improved detection and surveillance of infectious diseases in Kenya and other LMICs.

Finally, this category includes maternal and neonatal conditions. As a primarily adult, A\&E with a separate PEU, many neonatal conditions are evaluated in the PEU, such as lower respiratory illnesses and diarrheal diseases, which rank \#2 and \#3 for YLL in Kenya, respectively. ${ }^{43}$ Conducting a similar study in the PEU would provide a more comprehensive understanding of these paediatric conditions.

\section{Other (undifferentiated disease)}

Third category of 'other' consists of diagnoses and chief complaints that do not fit into the GBD categorisation. 
These are syndromic complaints such as 'abdominal pain', 'chest pain' and 'headache'. These complaints fall under 'symptoms, signs and abnormal clinical and laboratory findings, not elsewhere classified' which comprised the majority of our chief complaints (44\%). These undifferentiated complaints were better categorised into one of the three GBD categories as their final diagnoses were elucidated (undifferentiated final diagnoses made up 25\%). There is a need for specialised training for evaluating patients with undifferentiated and syndromic complaints, an area where EM training is well suited. ${ }^{44}$ The specialty of EM is by nature multidisciplinary, allowing for the rapid triage and treatment of acute injury or illness of all organ systems and populations. A proficiency in stabilising therapeutics and procedures acquired from a multitude of medical and surgical specialties, gives the EM-trained physician a decided advantage and efficiency for treating undifferentiated A\&E patients. Further, EM-trained physicians demonstrate improved consistency and quality of care in Emergency Departments. ${ }^{45-47}$ However, EM as a specialty, is not recognised by the Kenya Medical and Dentists Board (KMDB). EM development in Kenya starts with recognising EM as a specialty, which could allow residency-trained emergency physicians to form local faculty for training of healthcare providers in EM. ${ }^{6}$

\section{Study limitations}

The ratio of GBD categories presenting to KNH appears different than Kenya's country level burden of disease pattern. This could be due to the lack of paediatric data as our study does not include patients who presented to the separate PEU. The paediatric patients that we captured were skewed towards the critically ill and injured paediatric population. A study describing the population presenting to the PEU would certainly add to our body of knowledge in this area. This could also be due to seasonal variation in presentations. Finally, the $25 \%$ of final diagnoses that remain in the 'undifferentiated' category may also contribute to this difference.

As a tertiary care hospital, the burden of disease detected at KNH will be skewed towards higher acuity conditions than would be present in secondary or primary level centres with emergency care. Therefore, the generalisations of emergency care burden to the other hospital settings in LMICs should be made cautiously. However, the results of this study, combined with the findings at Moi Teaching and Referral Hospital provide an excellent snapshot of tertiary care epidemiology in Kenya. ${ }^{16}$ Future studies evaluating district and rural A\&Es, combined with our data, would provide an improved picture of the acute disease burden in Kenya.

A potential pitfall of using GBD categories to assess emergency care is the imprecision in grouping chief complaints and undifferentiated illness. The net effect, when comparing to other GBD studies, would be an underestimation of the burden of acute disease. Since chief complaints may better characterise acute disease,${ }^{44}$ it could be argued that future GBD should include variables to capture this data. ICD-10 is the international standard for categorising epidemiological and health management data and is used to classify diagnoses as well as reasons for consultation (chief complaint). ${ }^{17}$ However, concessions to better capture the ICD-10 category, 'symptoms, signs and abnormal clinical and laboratory findings, not elsewhere classified' would be valuable.

There were limitations due to current A\&E data systems. The A\&E is high volume with paper-based charting and minimal patient tracking. There are no centralised patient tracking boards or electronic tracking systems. There is variability in the extent of documentation among health providers. RAs had to personally follow their enrolled subjects throughout each shift. Our RAs noted that some patients bypassed triage and were not recorded in the triage book and therefore were not included in their systematic sampling. These missing data limit the accuracy of the disposition estimates. These challenges were not anticipated and will be taken into account in the design of future studies. Additionally, although it would be ideal to enrol every patient for the most accurate description of the total burden of disease, systems for a complete prospective registry in this setting are currently not available.

In addition, the requirement for an informed written consent for this registry project forced sampling bias, as critically ill patients could not be consented and therefore were excluded. This combined with difficulties tracking patient disposition, it is likely that actual admission and death rates are higher than our estimates. Based on our review of their admission logbooks and the total patient census, we estimate that $30 \%$ of patients typically are admitted, appreciably higher than our results. As long as written consent is required and due to the limitations of a prospective study design in this setting, it is likely that a retrospective study may yield more accurate data for certain statistics, such as admission rates. However, retrospective studies have other associated limitations and, given our experience, it is likely that in LMIC A\&Es, retrospective and prospective studies fulfil different roles.

\section{CONCLUSIONS}

This pilot study is a novel, prospective study characterising the A\&E population at a tertiary care centre in Kenya. This study highlights the growing burden of injuries and NCDs-compounding the existing burden of communicable conditions, presenting for acute care. The majority of patients are presenting with acute conditions related to NCDs and injuries. The prevalence of infectious disease is likely underreported by this study. Given large patient volumes, shifting epidemiology and the growing recognition of the importance of emergency care, a comprehensive understanding of the acute care needs at Kenya's largest referral centre is long overdue, both to guide patient care in the acute setting and to inform broader public health and policy agendas. Our findings provide an initial frame of reference in which to guide development of emergency resources and training at $\mathrm{KNH}$. 
Author affiliations

${ }^{1}$ Department of Emergency Medicine, University of North Carolina School of Medicine, Chapel Hill, North Carolina, USA

${ }^{2}$ Department of Emergency Medicine, The Ohio State University College of Medicine, Columbus, Ohio, USA

${ }^{3}$ Department of Emergency Medicine, Saint Joseph Hospital, Denver, Colorado, USA ${ }^{4}$ Accident and Emergency Department, Kenyatta National Hospital, Nairobi, Kenya ${ }^{5}$ Department of Orthopedics, School of Medicine, University of Nairobi, Nairobi, Kenya

${ }^{6}$ Department of Emergency Medicine, Hennepin County Medical Center, Minneapolis, Minnesota, USA

${ }^{7}$ Department of Emergency Medicine, School of Medicine, West Virginia University, Morgantown, West Virginia, USA

Acknowledgements The authors would like to thank the following people for their support and the success of this project: Dr Simeon Monda, Deputy Director of Clinical Services (Immediate Past Director); Drs Hassan Ibrahim and Linda Mose (Immediate past Heads of A\&E); Philomena Maina and Elizabeth Ndegwa, Heads of Nursing and Rachel Maina, Health Information Manager. In addition, we want to extend our gratitude to our research assistants for their hard work: Ritika Dhanda, Ruth Kara, Justin Francis, Emmanuel Keya, Tom Mathinji, Tatiana Mutinda, Collins Sudi, Rajvi Tewary, Yash Agrawal, Daniel Bacon, Wes Davis, John Sudor and Sarah Zamamiri. Thank you very much (Asante Sana) to the Departments of A\&E and Orthopedics at KNH for their support and contributions to this research endeavour.

Contributors JM was responsible for the conception of idea for study design, draft of protocol, data collection, analysis and manuscript initiation, reading and approval of final manuscript. KE prepared the RedCap Database. KH was responsible for the statistical analysis and key manuscript formatting. AW was instrumental in the development of the final protocol. KE, AW, AM and IM assisted with data collection. $\mathrm{KMH}, \mathrm{KE}, \mathrm{AW}, \mathrm{AM}, \mathrm{VM}, \mathrm{SD}$ and IBKM were responsible for the analysis and revisions of study design and protocol, analysis and critical revisions of manuscript. All authors had the opportunity to read and approve the final manuscript.

Funding Research funding was provided by the Global Health and Leadership Fellowship at the University of North Carolina and the International Emergency Medicine Fellowship at Hennepin County Medical Center (via the Hennepin Health Foundation)

Competing interests None declared.

Patient consent Obtained.

Ethics approval University of North Carolina Institutional Review Board and the University of Nairobi/Kenyatta National Hospital Ethics Review Committee.

Provenance and peer review Not commissioned; externally peer reviewed.

Data sharing statement The database is conjointly owned between the individual co-investigators and their respective departments, including the University of Nairobi and the Kenyatta National Hospital Accident and Emergency Department. The ethical approval for this study obliges us to follow our data sharing agreement (available for review) and to protect the privacy of participants. According to our agreement (filed through the UofN/KNH ERC in 2014-2015), requests for access to this database for data analysis from investigators or institutions outside of the owners will be considered, pending stipulations of Data Use Agreement are observed and that all owners are made aware. Readers can apply for access and permission to this database by contacting the project coordinator at justin_myers@ med.unc.edu or through contact with any of the co-investigators.

Open Access This is an Open Access article distributed in accordance with the Creative Commons Attribution Non Commercial (CC BY-NC 4.0) license, which permits others to distribute, remix, adapt, build upon this work non-commercially, and license their derivative works on different terms, provided the original work is properly cited and the use is non-commercial. See: http://creativecommons.org/ licenses/by-nc/4.0/

(c) Article author(s) (or their employer(s) unless otherwise stated in the text of the article) 2017. All rights reserved. No commercial use is permitted unless otherwise expressly granted.

\section{REFERENCES}

1. Marquez P V, Farrington JL. The challenge of non-communicable diseases and road trafficinjuries in Sub-Saharan Africa. An overview. 79293: Washington DC World Bank, 2013.
2. Lozano R, Naghavi M, Foreman K, et al. Global and regional mortality from 235 causes of death for 20 age groups in 1990 and 2010: a systematic analysis for the global burden of disease study 2010. Lancet 2012;380:2095-128.

3. Cox M, Shao J. Emergency medicine in a developing country: experience from Kilimanjaro Christian Medical Centre, Tanzania, East Africa. Emerg Med Australas 2007;19:470-5.

4. Razzak JA, Kellermann AL. Emergency medical care in developing countries: is it worthwhile? Bull World Health Organ 2002;80:900-5.

5. Hsia R, Razzak J, Tsai AC, et al. Placing emergency care on the global agenda. Ann Emerg Med 2010;56:142-9.

6. Wachira B, Martin IBK. The state of emergency care in the Republic of Kenya. Afr J Emer Med 2011;1:160-5.

7. Maher A, Sridhar D. Political priority in the global fight against noncommunicable diseases. J Glob Health 2012;2:20403.

8. Nugent R, Feigl AB. Where have all the donors gone? scarce donor funding for non-communicable diseases working paper 228. Cent Glob Dev 2010 http://hdl.handle.net/123456789/30109

9. Ravishankar N, Gubbins P, Cooley RJ, et al. Financing of global health: tracking development assistance for health from 1990 to 2007. Lancet 2009;373:2113-24.

10. Vu A, Duber HC, Sasser SM, et al. Emergency care research funding in the global health context: trends, priorities, and future directions. Acad Emerg Med 2013;20:1259-63.

11. Aufderheide TP, Nolan JP, Jacobs IG, et al. Global health and emergency care: a resuscitation research agenda - part 1. Acad Emerg Med 2013;20:1289-96.

12. Obermeyer Z, Abujaber S, Makar M, et al. Emergency care in 59 lowand middle-income countries: a systematic review. Bull World Health Organ 2015;93:577-86.

13. Harris PA, Taylor R, Thielke R, et al. Research electronic data capture (REDCap) - a metadata-driven methodology and workflow process for providing translational research informatics support. J Biomed Inform 2009;42:377-81.

14. University of Minnesota. Clinical and Translational Science Institute. REDCap is a secure web application designed to support data capture for research. 2016 http://www.ctsi.umn.edu/researcherresources/tools-and-software/redcap

15. World Health Orangization. International classification of diseases: online version, 2015. http://www.who.int/classifications/icd/en/

16. House DR, Nyabera SL, Yusi K, et al. Descriptive study of an emergency centre in Western Kenya: challenges and opportunities. Afr J Emerg Med 2014;4:19-24.

17. World Health Organization. International statistical classification of diseases and related health problems: instruction manual. 2, 2011. http://www.ncbi.nlm.nih.gov/pubmed/22184833

18. Department of Health Statistics and Information Systems WHO WHO methods and data sources for global burden of disease estimates 2000-2011. 2013 http://www.who.int/healthinfo/statistics/ GlobalDALYmethods_2000_2011.pdf?ua=1

19. Haagsma JA, Graetz N, Bolliger I, et al. The global burden of injury: incidence, mortality, disability-adjusted life years and time trends from the Global Burden of Disease study 2013. Inj Prev 2016;22:1-16.

20. Twomey M, Wallis LA, Thompson ML, et al. The South African triage scale (adult version) provides valid acuity ratings when used by doctors and enrolled nursing assistants. Afr J Emerg Med 2012;2:3-12.

21. Wachira BW, Wallis LA, Geduld H. An analysis of the clinical practice of emergency medicine in public emergency departments in Kenya. Emerg Med J 2012;29:473-6.

22. Wachira BW, Owuor AO, Otieno HA. Acute management of STelevation myocardial infarction in a tertiary hospital in Kenya: are we complying with practice guidelines? Afr J Med Med Sci 2014;4:104-8.

23. Echoka E, Kombe Y, Dubourg D, et al. Existence and functionality of emergency obstetric care services at district level in Kenya: theoretical coverage versus reality. BMC Health Serv Res 2013;13:113.

24. Levitt NS, Steyn K, Dave J, et al. Chronic noncommunicable diseases and HIV-AIDS on a collision course: relevance for health care delivery, particularly in low-resource settings - insights from South Africa. Am J Clin Nutr 2011;94:1690S-6.

25. .Institute for Health Metrics and Evaluation. Kenya. http://www. healthdata.org/kenya 2017.

26. WHO. Global status report on noncommunicable diseases, 2010.

27. Somanje H, Toure B, Drame B, et al. Optimizing global health initiatives to strengthen National Health Systems, 2009.

28. BBC. Zika outbreak: What you need to know. 2016 http://www.bbc. com/news/health-35370848 (accessed 4 Sep 2016). 
29. CNN. Complete coverage: the Ebola outbreak. 2016 http://www.cnn. com/specials/health/ebola (accessed 4 Sep 2016).

30. Young ME, Norman GR, Humphreys KR. Medicine in the popular press: the influence of the media on perceptions of disease. PLOS One 2008;3:e3552-7.

31. Hsia RY, Ozgediz D, Mutto M, et al. Epidemiology of injuries presenting to the national hospital in Kampala, Uganda: implications for research and policy. Int J Emerg Med 2010;3:165-72.

32. Hyder AA, Wunderlich CA, Puvanachandra P, et al. The impact of traumatic brain injuries: a global perspective. NeuroRehabilitation 2007;22:341-53.

33. De Silva MJ, Roberts I, Perel P, et al. Patient outcome after traumatic brain injury in high-, middle- and low-income countries: analysis of data on 8927 patients in 46 countries. Int J Epidemiol 2009;38:452-8.

34. Staton C, Vissoci J, Gong E, et al. Road traffic injury prevention initiatives: a systematic review and metasummary of effectiveness in low and middle income countries. PLOS One 2016;15.

35. Saidi H, Mutiso BK, Ogengo J. Mortality after road traffic crashes in a system with limited trauma data capability. J Trauma Manag Outcomes 2014;8:4.

36. WHO. Global status report on road safety 2013: supporting a decade of action. Geneva, 2013. www.who.int

37. World Health Organization. Road safety in 10 countries. Kenya, 2010. http://www.who.int/violence_injury_prevention/road_traffic/ countrywork/ken/en/

38. World Health Organization. Violence and injury prevention: road safety. Kenya: WHO, 2016. http://www.who.int/violence_injury_ prevention/road_traffic/countrywork/kenya/en/ (accessed 2 Sep 2016).

39. UNAIDS. Country factsheets KENYA | 2014 HIV and AIDS estimates, 2014. http://aidsinfo.unaids.org/ (accessed 1 Jan 2016).

40. Kelen GD, Hsieh YH, Rothman RE, et al. Improvements in the continuum of HIV care in an inner-city emergency department. AIDS 2016;30:113-20.

41. Kelen GD, Rothman RE. Emergency department-based HIV testing: too little, but not too late. Ann Emerg Med 2009;54:65-71.

42. Mohareb AM, Rothman RE, Hsieh YH. Emergency Department (ED) utilization by HIV-infected ED patients in the United States in 2009 and 2010 - a national estimation. HIV Med 2013;14:605-13.

43. IHME (Institute for Health Metrics and Evaluation). Kenya country facts. Global burden of disease study, 2016. http://www.healthdata. org/kenya (accessed 4 Sep 2016).

44. Mowafi $\mathrm{H}$, Dworkis D, Bisanzo M, et al. Making recording and analysis of chief complaint a priority for global emergency care research in low-income countries. Acad Emerg Med 2013;20:1241-5.

45. Weaver CS, Avery SJ, Brizendine EJ, et al. Impact of emergency medicine faculty on door to thrombolytic time. J Emerg Med 2004:26:279-83.

46. Jones JH, Weaver CS, Rusyniak DE, et al. Impact of emergency medicine faculty and an airway protocol on airway management. Acad Emerg Med 2002;9:1452-6.

47. McNamara RM, Kelly JJ. Impact of an emergency medicine residency program on the quality of care in an urban community hospital emergency department. Ann Emerg Med 1992;21:528-33. 